\title{
TV/Series
}

15 | 2019

La Sérialité en question(s)

\section{Comment étudier la complexité des séries télévisées ?: vers une approche spatiale}

\section{Mireille Berton et Marta Boni}

\section{Q OpenEdition}

1 Journals

Édition électronique

URL : http://journals.openedition.org/tvseries/3691

DOI : 10.4000/tvseries.3691

ISSN : 2266-0909

Éditeur

GRIC - Groupe de recherche Identités et Cultures

Référence électronique

Mireille Berton et Marta Boni, « Comment étudier la complexité des séries télévisées ? : vers une approche spatiale », TV/Series [En ligne], 15 | 2019, mis en ligne le 16 juillet 2019, consulté le 20 juillet 2019. URL : http://journals.openedition.org/tvseries/3691 ; DOI : 10.4000/tvseries.3691

Ce document a été généré automatiquement le 20 juillet 2019.

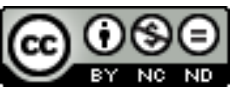

TV/Series est mis à disposition selon les termes de la licence Creative Commons Attribution - Pas d'Utilisation Commerciale - Pas de Modification 4.0 International. 


\title{
Comment étudier la complexité des séries télévisées? : vers une approche spatiale
}

\author{
Mireille Berton et Marta Boni
}

1 Comment étudier les spécificités des séries, ces récits évolutifs, multiformes, ouverts, articulés en plusieurs fragments? Aujourd'hui, non seulement les séries sont diffusées et se déploient à travers différents médias, mais elles sont également consommées selon des modalités variées, comme l'illustrent les franchises Marvel, pour lesquelles la sortie en salle de plusieurs films aux intrigues connexes s'allie à des séries télévisées diffusées sur les réseaux généralistes ou sur Netflix. Dans ce cadre de sérialisation élargie, la répétition et la nécessaire variation, typiques de la sérialité ${ }^{1}$, continuent d'attirer, de fidéliser et de surprendre les consommateurs.rices (qui sont à leur tour des producteurs.rices de contenu). Partant, ne faudrait-il pas envisager la sérialité comme un phénomène transversal qui s'inscrit dans l'histoire culturelle de différents médias ${ }^{2}$ - les mondes transmédiatiques ${ }^{3}$ étant des univers ou systèmes de contenu à partir desquels un répertoire d'histoires et de personnages fictifs peut être actualisé à travers plusieurs médias?

2 Sur la base de ce constat, nous pouvons mettre en évidence trois problèmes qui émergent pour les chercheurseuses qui s'emploient à les décrire :

1. le problème de la quantité (nous sommes dans le contexte d'un excès de données, d'une «situation d'offre pléthorique », pour reprendre les termes d'Yves (itton ${ }^{4}$ );

2. le problème de l'instabilité (les formes et les plateformes ne cessent de se réagencer);

3. le problème de la complexité (la série est un objet non-discret, ou continu, qui se transforme dans le temps selon une évolution qui, dans certains cas, est prévue par ses producteurs-rices, mais qui mérite aussi d'être pensée dans le cadre d'une porosité des frontières avec les différents domaines de la réception).

3 La question de la légitimité des séries télévisées a pendant longtemps dominé dans les premiers travaux qui revendiquent systématiquement le droit de s'intéresser à la culture populaire ${ }^{5}$, l'enjeu consistant à montrer qu'elles méritent de figurer au panthéon des 
œuvres « artistiques » (pensons à la place d'honneur qu'on leur accorde dans des festivals de cinéma comme Cannes ou Sundance ou encore dans des festivals spécifiquement consacrés à elles, comme Séries Mania à Lille). Or, si cette question n'est désormais plus d'actualité, d'autres interrogations apparaissent, relatives à la manière d'analyser ces objets et leur prolifération. Doit-on s'appuyer sur les outils provenant des disciplines qui traditionnellement explorent les productions artistiques, telles les études littéraires ou les études cinématographiques, ou est-il nécessaire d'en élaborer de nouveaux? Comment appréhender des objets extrêmement denses et réflexifs qui non seulement semblent prendre en charge le discours critique qui revient d'habitude aux analystes, aux critiques, aux historien-nne's, mais qui se nourrissent également des discours des fans et des appropriations variées qui circulent sur la Toile? Dans ce cadre, les outils des études cinématographiques ou littéraires sont-ils suffisants ou faut-il concevoir d'autres méthodes qui seraient en mesure de rendre compte du caractère multiforme de la fiction sérielle?

Dans leur «Préface. Les séries télé dans le monde. Échanges, déplacements et transpositions ", Sylvaine Bataille et Sarah Hatchuel évoquent la pertinence d'inventer une discipline appelée "études des séries télévisées " - question à laquelle elles répondent par la négative : "[...] créer une nouvelle discipline autonome serait, à nos yeux, se priver de perspectives, de méthodologies, d'angles d'approche spécifiques aux diverses disciplines à même de produire une recherche inédite, riche, variée et évolutive ${ }^{6}$ ». L'objet " série télévisée » peut en effet se définir à la fois comme une forme de récit sériel, un produit commercial, une œuvre d'art, un divertissement, une expérience de pensée, une pratique culturelle. D'où la nécessité de mobiliser les ressources de différentes approches puisées dans des champs disciplinaires variés, comme l'illustrent les travaux développés dans les domaines de la sociologie, des sciences de l'information et de la communication, des études culturelles, de l'économie politique, de la narratologie postclassique, des neurosciences, de la psychanalyse, de la philosophie, etc. Les études réalisées par Séverine Barthes ${ }^{7}$ et Barbara Laborde ${ }^{8}$ sur le territoire français mettent en évidence l'éventail très large de méthodes utilisées - Laborde soulignant la récurrence de travaux qui croisent les perspectives et les outils d'analyse, mais aussi l'absence de consensus et la difficulté de combiner les différentes disciplines qui s'y attachent.

Souscrivant à cette proposition d'interdisciplinarité, nous développons ici une discussion visant à comprendre comment plusieurs disciplines, à première vue éloignées, telles que la géographie et les études cinématographiques, ou les études littéraires et les humanités numériques, peuvent nous aider à étudier les séries. Plus largement, il s'agit d'offrir une réflexion sur la place de l'étude de ces objets dans les sciences humaines et sociales, en rappelant la nécessité de croiser les approches existantes ainsi que d'en inventer de nouvelles afin de s'adapter aux mutations incessantes du territoire dessiné par les séries télévisées.

Dans un premier temps, il s'agira d'aborder le problème conjoint de la quantité, de l'instabilité, de la complexité que pose les séries, afin, dans un second temps, de proposer une approche qui permet de se saisir de cet objet complexe, afin de discuter l'apport des humanités numériques. 


\section{Le problème de la quantité}

7 Étudier les séries télévisées relève toujours un peu du défi, en raison de l'hétérogénéité des corpus, de cette multiplicité des composantes et de leur étalement dans la médiasphère. Si l'objet « série télévisée » est difficile à étudier, c'est aussi en raison de la variété et de l'ampleur des corpus qui peuvent aller jusqu'à des centaines d'heures. Le premier constat concerne donc la quantité des objets audiovisuels qui ne cessent de proliférer. Comment en effet appréhender ces programmes à l'ère de la «Peak TV ", terme proposé en août 2015 par le directeur de la chaîne FX, John Landgraf, pour mettre en évidence l'abondance de l'offre, tous canaux de diffusion confondus ? De 2009 à 2017, le nombre de séries diffusées aux États-Unis serait passé d'environ 200 à presque 490, sans compter les productions internationales 9 . La multiplication des acteurs.rices sociaux engagés dans la production est en partie responsable de ce «pic» de production, signe d'une émulation créative mais aussi cause de pression croissante exercée sur les dirigeante-s. de l'industrie télévisuelle qui doivent rester compétitifs'ves. De nombreux journalistes soulignent les conséquences de cette abondance pour les usagers : elle finit par générer davantage d'angoisses que de plaisir, les amateurs craignant de passer à côté de la perle rare et de ne pouvoir participer aux conversations suscitées par la dernière série "phénomène ». Sur le plan de la recherche, nous sommes rapidement confrontée's à la difficulté d'appréhender de manière cohérente des corpus aussi importants - sauf à restreindre le matériau et à mener des études de cas ciblées permettant d'obtenir davantage de précisions et de nuances, et éventuellement de tracer des tendances au sein desquelles différentes séries analogues peuvent s'inscrire.

\section{Le problème de l'instabilité}

$8 \quad$ Le deuxième défi auquel nous devons faire face lorsque nous étudions les séries télévisées réside dans la mutation constante - et donc l'instabilité - des formes et des plateformes de la télévision, média qui n'a jamais eu d'identité fixe ${ }^{10}$, sans compter l'absence de critères stables pour définir ces multiples instances, spécialement dans un contexte transnational. Car la série peut être définie de différentes manières, en fonction du point de vue que l'on adopte sur elle, mais aussi en fonction du pôle que l'on retient pour la caractériser, qu'il s'agisse du pôle de la production, de la diffusion, de la réception ou de la consommation. Dans le milieu francophone, plusieurs termes ont été développés pour classer les séries relativement à leur organisation temporelle ${ }^{11}$, avec peu de correspondance avec les typologies bâties à l'étranger ${ }^{12}$. Cette pluralité de grilles de lecture atteste ainsi d'un manque de consensus sur les formes, les formats (le terme luimême est utilisé pour définir d'une part la "recette" immuable dans le cadre des importations transnationales par Moran et Chalaby entre autres et, d'autre part, la relation au temps et à la forme par Soulez ${ }^{13}$ ) et les «formules » des séries. Elle souligne aussi que l'intérêt principal des chercheurseuses réside dans la relation au temps, considérée comme essentielle pour saisir la variabilité et la richesse de ces objets, cette focalisation sur la dimension temporelle des séries s'expliquant peut-être par la fascination que suscitent les séries «téléologiques " analysées brillamment par Florent Favard $^{14}$. 
Étudier les séries télévisées exige en effet une grande réactivité face aux transformations des industries culturelles qui ne cessent d'adapter leurs politiques éditoriales en fonction d'impératifs dictés à la fois par le marché et le public. Pour répondre, par exemple, au phénomène de la « Peak TV » et ses effets de saturation, les chaînes tendent ces dernières années à réduire le nombre de nouvelles séries, proposant des programmes plus courts en termes de saisons et d'épisodes (dont les durées peuvent fluctuer), favorisant les projets de mini-séries ou de séries d'anthologie, ou alors évitent d'annuler trop rapidement une production sur la base de taux d'audience peu satisfaisants (puisqu'elle pourra toujours être vue après la fin de sa diffusion officielle sur d'autres plateformes et sous d'autres formats). Une autre stratégie consiste à étendre autant que possible la diffusion des séries à succès en insérant des pauses plus longues entre les saisons, comme c'est le cas pour Game of Thrones (HBO, 2011-2019) ou Westworld (HBO, 2016-).

Ces transformations sont confirmées par les travaux de Luca Barra ${ }^{15}$ sur le genre de la comédie américaine dont les stratégies narratives et d'implication des spectateurice's ont été progressivement modifiées suite à l'apparition de nouveaux modes de diffusion et de consommation encouragés par les plateformes de vidéos à la demande et de streaming. Alors que la comédie des années 1990 cherchait à intégrer lella spectateur:ice à travers le rire et la diffusion des épisodes en fonction du temps conjoncturel (les séries suivant étroitement le calendrier de la vie sociale), les nouvelles comédies comme Master of None (Netflix, 2015-) ou Atlanta (FX, 2016-) abandonnent partiellement le rire au profit des codes du drame et du mélodrame (elles deviennent des "dramedy »), et se composent, principalement lors des premières saisons, d'épisodes relativement autonomes traitant d'un sujet précis (souvent annoncé à travers le titre). Selon Barra, la multiplication des plateformes de production et de diffusion de séries, ainsi que la disparité des pratiques de visionnement sur des supports non télévisuels, mettent en effet en crise le principe de l'articulation étroite entre les séries et le quotidien de l'usager, les scénaristes et producteurs-rices étant alors obligée's de trouver des solutions pour s'assurer l'adhésion du public, tout en soutenant de nouvelles pratiques de consommation telle le «bingewatching ». La connexion avec le public passe alors par une réduction du principe de la répétition (central dans le genre de la comédie), une simplification des intrigues, un affaiblissement de l'alignement chronologique du récit au temps social, ainsi qu'une assimilation des codes du drame et du mélodrame favorables à l'attachement à l'univers fictionnel ${ }^{16}$.

\section{Le problème de la complexité (notamment) narrative}

11 Outre la quantité et l'instabilité de la production et de leurs conséquences respectives sur la réception, surgit le problème de l'excellence narrative et esthétique de certaines séries puisque tout y est totalement déconstruit et réfléchi : les stéréotypes sont à la fois présents et remis en question; les points de vue sont tous représentés et incarnés par différents personnages par ailleurs tous complexes et contradictoires; toutes les interprétations sont possibles, y compris les plus incompatibles entre elles. Il peut donc s'avérer difficile de procéder à un travail de déconstruction puisque celui-ci est inclus dans les séries elles-mêmes. Les fictions sérielles actuelles manifestent en effet un très haut degré de réflexivité qui en fait des objets à la fois complets et complexes, intégrant dans la diégèse, la rhétorique et le fonctionnement textuel leur propre interprétation, voire leur propre critique. Cette dimension métadiscursive peut présenter le risque de 
nous rendre captifs'ves de notre propre admiration, la «perfection » de ces objets étant susceptible d'inhiber en partie l'esprit critique.

Jason Mittell propose un cadre conceptuel pour distinguer différents types de récits sérialisants au sein du paradigme qu'il nomme la «Complex TV ». Celle-ci (à ne pas confondre avec la « Quality $\mathrm{TV}^{17} »$ ), désigne des séries qui combinent de manière variable les logiques épisodique et feuilletonnante et qui engagent pleinement le spectateur, invitant à une lecture concentrée et participative en raison de récits riches d'un point vue tant esthétique que narratif. Ce terme s'applique en particulier aux séries dramatiques à l'instar de The Wire (HBO, 2002-2008), Lost (ABC, 2004-2010), Mad Men (AMC, 2007-2015), Breaking Bad (AMC, 2008-2013), Game of Thrones, House of Cards (Netflix, 2013-2018) ou True Detective (HBO, 2014-), lesquelles sont jugées sophistiquées et nuancées, déployant une vision du monde complexe qui s'enrichit au fil des épisodes et des saisons. Ce type de complexité est caractérisé par des histoires qui ont une logique et une temporalité hautement articulées, souvent non-linéaires, à l'instar des «puzzle films ${ }^{18}$ » ou des « multidraft narratives ${ }^{19}$ ». Dans ces cas, le concept de «Complex TV » sert à définir un objet et les défis que celui-ci représente pour les spectateurice-s, rappelant la figure du cercle herméneutique qui exige de mettre constamment en relation les parties et le tout ${ }^{20}$. Aussi, les nombreux travaux consacrés à la question de la complexité narrative des séries télévisées envisagent régulièrement celles-ci sous le prisme de l'agencement complexe d'éléments sur un axe temporel, avec comme unité de base la dialectique entre innovation et répétition ${ }^{21}$.

13 La série allemande Dark (Netflix, 2017-) illustre bien le paradigme de la "Complex TV » qui repose sur une logique d'interconnexions à long terme entre les épisodes ${ }^{22}$, engageant les spectateurice's dans un jeu de pistes ou un "mind-game » hautement favorable à la tension narrative et à l'attente d'un dénouement sans cesse retardé23. Souvent comparée par les médias à Stranger Things (Netflix, 2016-), notamment en raison de son chronotope (petit village dans les années 1980), cette série qui compte actuellement deux saisons retrace l'histoire d'une suite de disparitions à travers le motif du voyage dans le temps. À l'ombre de tours de refroidissement d'une centrale nucléaire, convergent trois époques (2009, 1986 et 1953) et se croisent des personnages qui « prolifèrent ", à la manière de la logique mondaine ${ }^{24}$, pour habiter ces différentes strates temporelles. Pour s'orienter dans cette narration extrêmement touffue, lèla spectateurrice a tout intérêt à s'appuyer sur un arbre généalogique (facilement disponible sur le Net) qui permet de visualiser les liens formés par quatre familles sur trois générations. Comparée à un puzzle ou à un labyrinthe (lequel est d'ailleurs diégétisé), basée sur de nombreuses références philosophiques (Platon, Goethe, Nietzsche, Schopenhauer), cette série postule un type de spectateur.rice prêt à s'investir cognitivement et intellectuellement dans un récit exigeant, porté par une esthétique tout en clair-obscur et se développant dans le cadre d'une atmosphère pesante. Comme en témoigne nombre de cartes du temps de la série et même la fameuse encyclopédie collaborative Lostpedia décrite par Mittell (2015), des séries comme Lost avaient déjà emmené leurs spectateur.ice's très loin dans l'élaboration d'hypothèses permettant de percer le mystère de l'île où les rescapés d'un accident d'avion tentent de survivre. Mais si dans Lost les complexités narratives et les multiplications de flash-backs reflétaient parfois un manque d'inspiration des scénaristes qui ne savaient pas comment boucler certains arcs narratifs ${ }^{25}$, dans Dark, cet enchevêtrement de couches spatiotemporelles est très finement construit de manière à happer le public appelé à devenir des spectateurice's « hyper-cognitifs ${ }^{26}$ ». 
14 Néanmoins, nous ne pouvons pas penser la complexité des séries en termes purement narratifs. Que l'on pense par exemple aux campagnes des fans qui se proposent de sauver une série lorsque celle-ci est destinée à l'annulation, comme Sense8 (Netflix, 2015-2018) en 2017. Si elles sont des récits élargis comparables à des "écosystèmes narratifs ${ }^{27}$ ", elles étendent leurs frontières au-delà de leurs limites textuelles puisqu'elles se prolongent à travers différentes ramifications, formant ainsi un "monde » qui prime sur l'intrigue elle-même ${ }^{28}$; elles se prolongent notamment sur les réseaux sociaux, les blogs, les forums, les sites de fans, où elles suscitent des lectures et des commentaires qui viennent enrichir le récit premier - phénomènes qui peuvent être analysés à l'aune du concept de la transfictionnalitée ${ }^{2}$. Ainsi, le défi de la complexité concerne également la place de la série dans la médiasphère, $\mathrm{y}$ compris dans les traces transmédiatiques et les pratiques discursives générées par les usagers.

\section{Les séries et l'approche spatiale}

L'analyse de l'objet "série télévisée » ne peut donc faire l'économie de sa dimension élargie, et ce sur l'axe tant de la quantité que de la complexité. Mais sur la base de quelles approches ou de quels outils s'en saisir? Elle exige sans aucun doute de prendre en compte un certain nombre de paramètres spécifiques à cet objet, tel le principe du récit sériel susceptible de se développer à l'infini ${ }^{30}$, ou encore la dimension économique d'une «œuvre » qui est aussi un produit marchand ${ }^{31}$. Nous devons ajouter à cela la dimension spatiale de la série télévisée, présente à la fois dans la diégèse et dans les interactions entre les usagers, l'univers fictionnel et la sphère de la production ${ }^{32}$.

Comme le rappelle Letourneux, l'analyse des récits sériels - qui dominent désormais notre culture et notre quotidien - exige de forger des modalités d'interprétation originales car ce type de fiction rend caduques les notions d'auteur, d'œuvre et de légitimité artistique ${ }^{33}$. C'est pourquoi, il faut éviter d'« appréhender ces productions à partir des grilles analytiques de la culture légitimée, cherchant à mettre en avant de grands auteurs de genre, des œuvres sérielles originales, des visions du monde spécifiques. [.... $]^{34} »$. Letourneux invite ainsi à repenser la sérialité de sorte à se départir des modèles d'analyse qui ont prévalu jusqu'ici. Pour nous, il s'agit de trouver une alternative aux travaux qui tendent à se focaliser sur la progression temporelle d'un récit sériel, abordant principalement la question du nombre d'épisodes et de saisons en termes d'organisation narrative, à l'instar de Jean-Pierre Esquenazi qui appréhende la série télévisée comme un art $d u$ temps ${ }^{35}$. Or, la série peut aussi être saisie comme un art de l'espace, la consommation de fictions plurielles étant également faite d'interruptions (entre les épisodes, entre les saisons, entre une série et sa résurrection), lesquelles ne sont pas seulement des intervalles temporels justifiés par la narration mais aussi des espaces interstitiels, des territoires satellitaires avec lesquels toute écriture sérielle doit composer - et qui sont, par ailleurs, enrichis par des discours de spectateurice's et par d'autres paratextes. Ceci nous encourage donc à envisager la série, en complément de la perspective temporelle, dans sa dimension d'organisation spatiale.

17 Les récits sériels sont également bien plus que l'expression d'une culture de masse industrialisée qui produit des fictions standardisées, car ce point de vue tend à ignorer "l'hétérogénéité des pratiques, des positions auctoriales et des significations que les textes peuvent prendre une fois qu'ils ont été investis par les différents groupes sociaux ${ }^{36}$ ». L'analyse des productions sérielles doit idéalement englober l'analyse du contexte de 
production (ce que Letourneux nomme les « dynamiques architextuelles » en extrapolant la notion de Genette à l'origine peu sensible aux éléments contextuels) et l'analyse des textes (la micro-analyse au plan stylistique, narrative, esthétique, sémantique, etc.). Il semble de fait impossible d'envisager Game of Thrones sans prendre en compte son inscription dans l'architexte formé par la chaîne HBO qui en détermine fortement le contenu, notamment en codifiant la série comme de la «Quality TV »" En outre, la série est également à lire en termes de transcendance ${ }^{38}$ car comparable à une base de données, elle constitue une des possibilités incarnée, immanente, d'un monde plus large, transmédiatique et sous-jacent. Ainsi, la série télévisée peut être considérée en termes d'espace, dans la mesure où elle suggère un espace plus large qui la contient et la dépasse, rendant visible une portion de cet ensemble de données.

Il s'avère important de dépasser la sphère de l'intertexte pour cerner le contexte économique dans lequel apparaît une telle série ainsi que le milieu et les effets de sa réception. Car en tant que mode de communication reposant sur une "dynamique contradictoire " (à savoir, la fameuse tension entre reprise et variation), le récit sériel impose de renouveler nos méthodes d'analyse en considérant un ensemble de paramètres, à commencer par «la dimension collective et sociale des discours produits par les œuvres sériels, qui en fait l'un des espaces où s'expriment les positions idéologiques d'une époque et les tensions qui animent l'espace public ${ }^{39}$ ». En tant que lieux $d^{\prime}$ ' affrontements idéologiques, sociaux et esthétiques ${ }^{40} »$, les séries demandent donc de combiner l'analyse textuelle avec les problématiques historiques, médiatiques, sociales, économiques et politiques. Rappelons que, selon Jean-Pierre Esquenazi, il faut considérer l'œuvre « non comme un objet, mais comme un processus historique engendré dans un milieu social caractéristique et capable de prendre place dans différents milieux et situations ${ }^{41}$ ». Pour ces raisons, la prise en compte des pratiques d'appropriation des fans et, plus largement, des pratiques discursives, s'avère indispensable. Si le texte est, par étymologie, un réseau, à l'analyse de sa complexité interne il faut associer l'étude des supports qui en permettent la propagation dans le temps et dans l'espace.

C'est pourquoi, sur la base de la pensée d'Edgar Morin ${ }^{42}$, nous proposons d'adopter le modèle du système complexe de sorte à déplacer le centre d'attention de l'objet textuel (certes complexe du point de vue narratif) vers l'étude des interactions entre celui-ci et son contexte de production et de réception. Il s'agit en particulier de traiter les traces laissées par les usagers des séries télévisées, à savoir les artéfacts qui composent et accompagnent l'expérience de visionnage de tout produit audiovisuel et qui, de manière fondamentale pour un récit sériel, remplissent les espaces qui séparent la diffusion des épisodes, des saisons, des séries. Le système complexe englobe, organise le matériel et devient le terrain sur lequel peuvent surgir de nouvelles pratiques et de nouvelles significations, ainsi que de nouvelles orientations narratives : c'est un modèle qui offre des solutions à l'aporie qui s'impose lorsque nous voulons analyser l'espace se situant entre les textes et les spectateur.ice's.

Les spectateur.ice's sont en effet des générateurs.rices de traces résultant de nombreuses pratiques (narratives et non-narratives) qui vont s'ajouter au tissu hétérogène d'un récit complexe, lequel consolide également son identité par le biais du partage en réseau. Si la fragmentation et la multiplicité des données déterminent fortement la diffusion des séries télévisées, il y a néanmoins des réponses à chercher en termes de continuité car chaque élément relatif à un récit sériel s'inscrit dans un système en expansion continue. Nous proposons ainsi de décrire la série comme une sémiosphère ${ }^{43}$, à savoir un espace 
sémiotique doté d'une mémoire à long terme et ce, non seulement dans ses substructures, mais aussi en qualité de système complexe. Car chaque élément ne peut être pensé en dehors de son articulation avec les autres éléments du système ou de l'espace discursif plus large qui le contient. En termes sémiotiques, les divers fragments médiatiques trouvent une forme d'unité ou d'identité dans la sémiosphère qu'ils composent, laquelle constitue aussi la condition de leur existence. La série est donc un objet «à la fois inégal et pourtant unifié, asymétrique et pourtant uniforme ${ }^{44} »$. Cette conception de la série télévisée comme système sémiotique complexe au sein duquel convergent les traces d'une multitude de discours nous encourage à adopter une méthodologie spatiale de la série, en faisant appel aux instruments offerts par les humanités numériques et en utilisant en notre faveur le principe de multiplicité.

\section{L'apport des humanités numériques}

21 La série est un ensemble de données qui peuvent être récoltées, comparées et analysées à plusieurs niveaux. Des projets nés au croisement des études télévisuelles et des humanités numériques utilisent la quantification de la durée des plans ou des algorithmes de reconnaissance des visages pour analyser la répétition et la variation des formes à l'intérieur d'une série (comme par exemple le projet «Shot Logger» de Jeremy Butler) ${ }^{45}$. Par ailleurs, la proposition récente de certains chercheurseuses américaine's (parmi lesquelle·s, de manière intéressante, Jason Mittell) consiste à se tourner vers le deformative criticism $^{46}$, à savoir une façon de visualiser la série comme ensemble de plans qu'un logiciel permet de superposer, ou de chercher, via des algorithmes, des réponses concernant sa forme plutôt que sa dimension narrative. Si le temps demeure ici un concept-clé (la durée étant une catégorie centrale dans ces travaux), la tendance actuelle consiste davantage à chercher des voies qui s'éloignent des études basées sur la narratologie ou sur l'analyse des représentations. Cernée à partir de l'ensemble d'informations qu'elle contient, la série est toujours plus appréhendée comme un agglomérat de données.

Suivant le modèle importé de la sémiotique de la culture et de la relation de la série à l'espace, d'autres solutions méthodologiques, passant par les descriptions et les métadiscours entourant les séries, peuvent être envisagées. Il s'agit par exemple de se tourner vers la géographie et de prendre comme objet la série telle qu'elle apparaît dans l'espace géographique réel. Les recherches menées dans le cadre du Labo Télée ${ }^{77}$ proposent de chercher les titres de séries contenus dans l'Internet Movie Database (IMDb) et de les situer sur une carte, afin de restituer une cartographie du panorama sériel global (tel que contenu dans l'IMDb, donc certainement soumis à des biais d'hégémonie occidentale) qui permettra ensuite d'élaborer des questions concernant le nombre d'épisodes, les professionnels impliqués, les genres des séries, et ceci toujours en termes de localisation dans l'espace réel. Des remarques sur la récurrence de lieux de tournage, ou des pays producteurs et importateurs sont donc possibles via cette carte interactive qui devient aussi un moteur de recherche. Basée sur la spatialisation de l'analyse de catégories formelles et industrielles, cette approche s'inspire des travaux de Franco Moretti en littérature : dans ce cas, une «lecture distante » de corpus très larges permet de faire émerger des constantes et des variations dans les formes et dans les modes de production, sans passer par le traditionnel "close reading" auquel Moretti associe des biais de subjectivité et de légitimité culturelle ${ }^{48}$. 

d'épouser un point de vue qui soit respectueux des transformations historiques plus lentes et microscopiques et, par-delà, de restituer une vision "darwinienne" des mutations structurales du corpus, comme le proposent par exemple les chercheurseuses du groupe Narrative Ecosystems ${ }^{49}$. Cependant, si la position de Moretti reste marquée par un certain positivisme structuraliste, les cartes peuvent révéler autre chose que les relations géopolitiques dépendant des discours officiels car, conformément à l'image de la sémiosphère décrite plus haut, elles renseignent aussi sur l'existence de discours alternatifs ancrés dans la vie quotidienne des usagers.

À ce propos, nous avons montré, à travers une analyse de la réception sur Twitter et autres réseaux sociaux de Twin Peaks. The Return (Showtime, 2017) que l'outil des cartes peut informer sur plusieurs aspects de la vie d'une série, entendue en termes spatiaux ou de sémiosphère ${ }^{50}$. Par le biais de logiciels en libre accès comme OneMillionTweetMap, nous avons pu, par exemple, rendre visible la localisation des discours des fans : au lieu de mesurer en termes purement statistiques le nombre de contenus partagés par les fans ou les tweets contenant le hashtag sélectionné, nous avons pu voir, grâce aux cartes, l'intensité avec laquelle une série occupe une certaine place dans l'espace géographique global. L'intensité est déterminée par une plus grande densité des traces de discours dans un lieu précis ou à un moment déterminé, ce qui correspond par exemple à des Premières ou des Finales de saison, et nous renseigne sur la synchronie de la réception et des usages - ceci est d'autant plus intéressant que cela se produit malgré la possibilité, offerte dans le contexte de la télévision Post Network, de choisir à son gré quand et où visionner. Ces visualisations peuvent également devenir des points de départ pour une analyse des pratiques de piratage ou de contournement des dispositifs de geoblocking ${ }^{51}$. La série devient ainsi un forum culturel car si ce terme a été autrefois pertinent pour penser la télévision généraliste envisagée à la hauteur d'un pays entier, à l'ère actuelle des plateformes numériques, il peut également s'appliquer à des nombreuses niches de l'espace public. Aussi, potentiellement, chacune de ces traces pourra-t-elle encourager de nouvelles formes de consommation, fonctionnant comme une porte d'accès vers le monde de la série.

Si nous reprenons l'image de la sémiosphère, ces zones de participation plus intense correspondent à des pulsations dans le corps de la série : c'est là où "quelque chose se passe " et c'est là que des nouvelles questions peuvent surgir - en prenant garde, toutefois, de ne pas céder une forme de déterminisme ou de volonté prédictive. Les séries sont aussi des lieux où des interférences se produisent, correspondant à la rencontre - et donc à la nécessaire réaction adaptative - avec du matériel extratextuel (d'autres séries, d'autres médias), comme dans le cas des phénomènes transfictionnels. L'espace sériel est ainsi composé de situations microscopiques et de structures macroscopiques: les formes émergent à partir de mutations très lentes, de luttes et de frictions, mais aussi de convergences, à l'intérieur du panorama global des médias et à l'intérieur de ces sémiosphères que sont les séries, organismes vivants dans lesquels des associations inattendues entre les différentes parties sont possibles.

À partir de ce cadre, et par le biais d'instruments provenant du dialogue entre différentes disciplines, nous pouvons décrire les enjeux d'une approche basée sur les interférences entre formes et plateformes, entre sphère du texte officiel et sphère de la réception, avec l'objectif de trouver des réponses ou des éléments de réponse concernant les usages, l'esthétique et la narration des séries. D'une part, le modèle proposé doit nécessairement

TV/Series, 15 | 2019 
composer avec des questions posées préalablement ; d'autre part, des occurrences ou des résultats non prévisibles à l'avance peuvent surgir. L'analyse se base en effet sur les traces de la réception à partir de mots-clés choisis en fonction d'une connaissance préalable des modes de discursivité en ligne, ainsi que sur une série d'hypothèses sur la série et une connaissance approfondie de son contexte de production et de diffusion: dans le cadre de Twin Peaks, il s'agissait de chercher des réponses sur son statut culte, déjà confirmé par plusieurs études de l'œuvre et de la réception. Mais au-delà, et tenant compte de ces prémisses, le modèle proposé nous encourage à abandonner une analyse basée sur la discrétion (la série comme objet dont les frontières seraient établies à l'avance par des contraintes textuelles imposées par les producteurstrices officielllles) et à penser au contraire la série comme un espace continu, dans lequel se superposent et se croisent plusieurs éléments hétérogènes. De nouvelles questions sont alors à même de surgir du côté de l'espace situé entre les textes et les spectateur.ice's.

\section{Conclusion}

La série est donc analysable au niveau de sa spatialité, et à partir d'une certaine distance, et ce en raison de sa prolifération sur plusieurs territoires ainsi que de l'accessibilité possible à des traces d'interprétation qui ne cessent de se superposer, ces données permettant de visualiser ses dimensions tant quantitatives, formelles que technologiques. Susceptible de cerner l'objet sériel dans sa complexité narrative et non-narrative, l'approche spatiale favorise au final un phénomène qui parcourt et sature l'ensemble du panorama médiatique actuel: si Umberto Eco considérait la dialectique entre répétition et innovation comme étant centrale à la temporalité sérielle (et plus largement à l'esthétique postmoderne), penser la série télévisée en termes d'espace permet de l'appréhender à travers les principes à la fois de la disponibilité constante d'éléments transmédiatiques et de l'auctorialité multiple qui le composent, à l'image d'une base de données, instrument (et métaphore) indissociable de notre époque numérique. Il est donc temps de trouver et d'expérimenter de nouveaux outils et modèles conceptuels qui seraient en mesure de caractériser la sérialité télévisuelle dans ses coordonnées tant temporelles que spatiales, et de nous aider, à l'instar de Carrie Mathison tapissant minutieusement de post-its le mur de son appartement dans Homeland (Showtime, 2011-), à mieux voir les liens et les nœuds d'un système complexe, à travers son organisation spatiale.

\section{BIBLIOGRAPHIE}

ALLEN, Robert C. (dir.), To Be Continued... Soap Operas Around the World, Londres, New York, Routledge, 1995.

BARONI, Raphaël, La Tension narrative. Suspense, curiosité, surprise, Paris, Seuil, 2007. 
BARONI, Raphaël, JOST François (dir.), « Repenser le récit avec les séries télévisées », Télévision, n ${ }^{\circ}$ 7, 2016 : https://www.cairn.info/revue-television-2016-1.htm, consulté le 7 juillet 2019.

BARRA, Luca, « Master of None, Atlanta, and Audience Engagement in Contemporary American TV Comedy ", 16:9. Filmtidsskrift, 19 novembre 2017 : http://www.16-9.dk/2017/11/master-of-none/, consulté le 7 juillet 2019.

BARRA, Luca, « Les formes, durées et formats des comédies télévisuelles américaines.

Distribution, engagement du public et instabilité des frontières de genre », in BONI, Marta (dir.), Formes et plateformes de la télévision à l'ère du numérique, Rennes, Presses Universitaires de Rennes, à paraître en 2019.

BARTHES, Séverine, « Panorama de la recherche universitaire sur les séries télévisées en France ", Premières Rencontres des Séries Télévisées, France, 2004 : http://hal.archives-ouvertes.fr/ hal-00470237 (consulté le 7 juillet 2019).

BATAILLE, Sylvaine, HATCHUEL Sarah, «Préface. Les séries télévisées dans le monde : échanges, déplacements et transpositions »,TV/Series, $n^{\circ} 2,2012$, mis en ligne le 01 novembre 2012 : http:// journals.openedition.org/tvseries/1351, consulté le 7 juillet 2019.

BENASSI, Stéphane, Séries et feuilletons T.V. : pour une typologie des fictions télévisuelles, Liège, Éditions du CEFAL, 2000.

BENASSI, Stéphane, «Transfictions », MédiaMorphoses, Hors-Série, « Les raisons d'aimer les séries télé », Paris, Armand Colin, 2007, p. 158-162.

BERTON, Mireille, « Flashback, trauma et répétition narrative dans les séries américaines contemporaines », Télévision, $\mathrm{n}^{\circ}$ 7, 2016, p. 173-189.

BESSON, Anne, Constellations. Des mondes fictionnels dans l'imaginaire contemporain, Paris, CNRS Édition, 2015.

BESSON, Anne, « De l'ensemble à la totalité : l'effet de monde dans les littératures de l'imaginaire contemporaines », Belphégor, n 14, 2016 : http://journals.openedition.org/belphegor/650, consulté le 7 juillet 2019.

BOILLAT, Alain, Cinéma, machine à mondes : essai sur les films à univers multiples, Genève, Georg, 2014.

BONI, Marta, Romanzo Criminale. Transmedia and Beyond, Venise, Ca' Foscari Online Publishing, 2013, http://virgo.unive.it/ecf-workflow/upload_pdf/Innesti_2.pdf.

BONI, Marta, « Mondes sériels et complexité », Écrans, 2015, Volume 2, n 4, p. 55-68.

BONI, Marta, « Working with Maps: Systems, Networks, Series », in CAVALLOTTI, Diego, GIORDANO Federico, QUARESIMA, Leonardo (dir.), A History of Cinema Without Names II. A Research Project, FilmForum/2015, Udine, Mimesis, 2017, p. 69-76.

BONI, Marta (dir.), World Building. Transmedia, Fans, Industries, Amsterdam, Amsterdam University Press, 2017.

BONI, Marta, « Des téléspectateurs géolocalisés ». Sens Public, 15 décembre 2017 : http:// www.sens-public.org/article1283.html, consulté le 7 juillet 2019.

BORDWELL, David, « What-if Movies: Forking Paths in the Drawing Room », Observations on film art (blog), 2014: http://www.davidbordwell.net/blog/2014/11/23/what-if-movies-forking-paths-inthe-drawing-room/, consulté le 7 juillet 2019. 
BUCKLAND, Warren (dir.), Puzzle Films: Complex Storytelling in Contemporary World Cinema, Malden, Wiley-Blackwell, 2009.

BUONANNO, Milly, Le formule del racconto televisivo. La sovversione del tempo nelle narrative seriali, Florence, Sansoni, 2002.

BUXTON, David, Les séries télévisées. Formes, idéologie et mode de production, Paris, L'Harmattan, 2010.

CARDINI, Daniela, « Serial Contradictions: The Italian Debate on TV Series », Series. International Journal of TV Serial Narratives, Volume II, $\mathrm{n}^{\circ}$ 1, printemps 2016, p. 47-54: https://series.unibo.it/ article/view/6163, consulté le 7 juillet 2019.

CARDINI, Daniela, « Il tele-cinefilo. Il nuovo spettatore della Grande Serialità televisiva », Between. Rivista dell'Associazione di Teoria e Storia Comparata della Letteratura, Volume 4, n 8, novembre 2014, p. 1-30 : http://ojs.unica.it/index.php/between/article/view/1361?

fbclid =IwAR01riiC45RrKUXLIl4lT_Gv0YlPmPclEneLMrMEa1mbG8CmOkh_QkrQ9jY, consulté le 7 juillet 2019 .

CAUGHIE, John, « Telephilia and Distraction: Terms of Engagement », Journal of British Cinema and Television, Volume 3, $\mathrm{n}^{\circ} 1$, janvier 2008, p. 5-18.

CITTON, Yves, Pour une écologie de l'attention, Paris, Seuil, coll. « La Couleur des idées », 2014.

CHALABY, Jean, The Format Age : Television's Entertainment Revolution, Cambridge, Polity, 2015.

COLONNA, Vincent, L'art des séries télé, Paris, Payot, 2015.

DE KOSNIK, Abigail, « Piracy is the Future of Television », Convergence Culture Consortium, 2018, http://convergenceculture.org/research/c3-piracy_future_television-full.pdf, consulté le 7 juillet 2019.

ECO, Umberto, « Innovation et répétition : entre esthétique moderne et post-moderne », Réseaux, $\mathrm{n}^{\circ} 68,1994$, p. 1-18.

ESQUENAZI, Jean-Pierre, Les séries télévisées : l'avenir du cinéma ?, Paris, Armand Colin, 2007a.

ESQUENAZI, Jean-Pierre, Sociologie des cuvres : de la production à l'interprétation, Paris, Armand Colin, 2007b.

ESQUENAZI, Jean-Pierre, Éléments pour l'analyse des séries télévisées, Paris, L'Harmattan, 2017.

FAVARD, Florent, La promesse d'un dénouement. Énigmes, quêtes et voyages dans le temps dans les séries télévisées de science-fiction contemporaine, thèse de doctorat, Université Bordeaux Montaigne, 2015.

FAVARD, Florent, Le récit dans les séries de Science-fiction de Star Trek à X-files, Paris, Armand Colin, 2018.

FEUER, Jane, « HBO and the Concept of Quality TV », dans Janet MacCabe et Kim Akass (dir.), Quality TV: Contemporary American Television and Beyond, Londres, I.B. Tauris, 2007, p. 145-157.

GLEVAREC, Hervé, La sériephilie. Sociologie d'un attachement culturel, Paris, Ellipses, 2012.

GOUDMAND, Anaïs, « Narratologie du récit sériel. Présentation de quelques enjeux méthodologiques », Proteus [en ligne], n 6, 2013, p. 81-89 : http://www.revue-proteus.com/ abstracts/06-10.html, consulté le 7 juillet 2019.

GOUDMAND, Anaïs, « Les séries transmédiatiques. Des univers sans fins ? ", Proteus, $\mathrm{n}^{\circ}$ 9, 2015, p. 8-18 : http://www.revue-proteus.com/articles/Proteus09-1.pdf, consulté le 7 juillet 2019. 
HVEN, Steffen, Cinema and Narrative Complexity. Embodying the Fabula, Amsterdam, Amsterdam University Press, 2017.

INNOCENTI, Veronica, PESCATORE, Guglielmo, « Changing Series: Narratives Models and the Role of the Viewer in Contemporary Television Seriality ", Between. Rivista dell'Associazione di Teoria e Storia Comparata della Letteratura, Volume 4, nº 8, 2014, p. 1-15 : http://ojs.unica.it/index.php/ between/article/view/4, consulté le 7 juillet 2019 .

JENKINS, Henry, La culture de la convergence : des médias au transmédia, Paris, Armand Colin, 2013.

JENKINS, Henry, « Transmedia Education: the 7 Principles Revisited », Confessions of an Aca-fan (blog): http://henryjenkins.org/blog/2010/06/transmedia_education_the_7_pri.html, consulté le 7 juillet 2019.

LABORDE, Barbara, « Television Series: Inventory of Research in France », Series. International Journal of TV Serial Narratives, Volume III, n² 2, hiver 2017 : https://series.unibo.it/article/ view/7631, consulté le 7 juillet 2019.

LETOURNEUX, Matthieu, Fictions à la chaîne. Littératures sérielles et culture médiatique, Paris, Seuil, 2017.

LETOURNEUX, Matthieu (dir.), « Sérialités », Belphégor, n 14, 2016 : http:// journals.openedition.org/belphegor/647, consulté le 7 juillet 2019.

LOTMAN, Yuri, Universe of the Mind: A Semiotic Theory of Culture, Bloomington, Indiana University Press, 1990.

MICHLIN, Monica, « More, More, More. Contemporary American TV Series and the Attractions and Challenges of Serialization As Ongoing Narrative ", Mise au point, $\mathrm{n}^{\circ} 3$, 2011: http:// map.revues.org/927, consulté le 7 juillet 2019.

MORETTI, Franco, Atlas of the European Novel, 1800-1900, Londres, Verso, 1999.

MORETTI, Franco, « Planet Hollywood », New Left Review, n 9, mai-juin 2001 : https:// newleftreview.org/II/9/franco-moretti-planet-hollywood, consulté le 7 juillet 2019.

MORETTI, Franco, Graphs, Maps, Trees: Abstract Models for a Literary History, Londres, Verso, 2005.

MITTELL, Jason, Complex TV: The Poetics of Contemporary Storytelling, New York, NYU Press, 2015.

MORAN, Albert, Copycat Television: Globalisation, Program Formats and Cultural Identiy, Luton, Bedfordshire, UK, University of Luton Press, 1998.

MORIN, Edgar, Introduction à la pensée complexe, Paris, Seuil, 2005.

RE, Valentina, « Beyond the Threshold. Paratext, Transcendence, and Time in the Contemporary Media Landscape », in NOTO, Paolo, PESCE, Sara (dir.), The Politics of Ephemeral Digital Media: Permanence and Obsolescence in Paratexts, New York, Londres, Routledge, 2016, p. 60-74.

RODRIGUEZ, Ashley, « The End of Peak TV Must Finally, Mercifully Be Nigh », Quartz, 13 janvier 2018: https://qz.com/999827/the-end-of-peak-tv-must-finally-mercifully-be-nigh/, consulté le 7 juillet 2019.

SAINT-GELAIS, Richard, Fictions transfuges. La transfictionnalité et ses enjeux, Paris, Seuil, 2011.

SOULEZ, Guillaume, KITSOPANIDOU, Kira (dir.), « Le levain des médias - Forme, format, média ». MEI - Médiation et information, $\mathrm{n}^{\circ}$ 39, Paris, L'Harmattan, 2015. 
URICCHIO, William, « Television's Next Generation: Technology/Interface Culture/Flow », in SPIGEL, Lynn, OLSSON, Jan (dir.), Television After TV. Essays on a Medium in Transition, Durham, Londres, Duke University Press, 2004, p. 163-182.

WINCKLER, Martin, Petit éloge des séries télé, Paris, Gallimard, 2012.

\section{NOTES}

1. Eco Umberto, «Innovation et répétition : entre esthétique moderne et post-moderne », Réseaux , $\mathrm{n}^{\circ} 68,1994$, p. 1-18.

2. En ce sens, il s'agirait d'accorder une place centrale à l'un des sept principes identifiés par Henry Jenkins comme fondateurs du "transmedia storytelling ", à savoir la sérialité. Voir son blog, Confessions of an Aca-fan: http://henryjenkins.org/blog/2010/06/ transmedia_education_the_7_pri.html, consulté le 7 juillet 2019.

3. Marta Boni, Romanzo Criminale. Transmedia and Beyond, Venise, Ca' Foscari Online Publishing, 2013, http://virgo.unive.it/ecf-workflow/upload_pdf/Innesti_2.pdf; Marta Boni (dir.) , World Building. Transmedia, Fans, Industries, Amsterdam, Amsterdam University Press, 2017.

4. Yves Citton, Pour une écologie de l'attention, Paris, Seuil, coll. « La Couleur des idées », 2014.

5. Jean-Pierre Esquenazi, Les séries télévisées : l'avenir du cinéma?, Paris, Armand Colin, 2007 ; Martin Winckler, Petit éloge des séries télé, Paris, Gallimard, 2012 ; Vincent Colonna, L'art des séries télé, Paris, Payot, 2015.

6. Sylvaine Bataille et Sarah Hatchuel, «Préface. Les séries télévisées dans le monde : échanges, déplacements et transpositions ", TV/Series, $\mathrm{n}^{\circ} 2$, 2012, mis en ligne le 01 novembre 2012 : http:// journals.openedition.org/tvseries/1351, consulté le 12 mars 2018.

7. Séverine Barthes, «Panorama de la recherche universitaire sur les séries télévisées en France », Premières Rencontres des Séries Télévisées, France, 2004 : http://hal.archives-ouvertes.fr/ hal-00470237, consulté le 7 juillet 2019.

8. Barbara Laborde, "Television Series: Inventory of Research in France », Series. International Journal of TV Serial Narratives, Volume III, $\mathrm{n}^{\circ} 2$, hiver 2017: https://series.unibo.it/article/ view/7631, consulté le 7 juillet 2019.

9. Ashley Rodriguez, «The End of Peak TV Must Finally, Mercifully Be Nigh », Quartz, 13 janvier 2018: https://qz.com/999827/the-end-of-peak-tv-must-finally-mercifully-be-nigh/, consulté le 7 juillet 2019.

10. William Uricchio, "Television's Next Generation: Technology/Interface Culture/Flow ", in Television After TV. Essays on a Medium in Transition, éd. Lynn Spigel et Jan Olsson, Durham, Londres, Duke University Press, 2004, p. 163-182.

11. Stéphane Benassi, Séries et feuilletons T.V.: pour une typologie des fictions télévisuelles, Liège, Éditions du CEFAL, 2000 ; Esquenazi, op.cit.. Le 28 février 2018, Claire Cornillon proposait à l'Université Le Havre Normandie une communication sur la sérialité semi-feuilletonnante dans laquelle les termes utilisés par Jean-Pierre Esquenazi étaient utilisés pour décrire des objets très différents, voire opposés : https://www.youtube.com/watch? $\mathrm{t}=202 \mathrm{~s} \& \mathrm{v}=\mathrm{X} 3 \mathrm{NT}_{-} 4 \mathrm{oYjDw} \&$ app $=$ desktop.

12. Milly Buonanno, Le formule del racconto televisivo. La sovversione del tempo nelle narrative seriali, Florence, Sansoni, 2002; Jason Mittell, Complex TV. The Poetics of Contemporary Storytelling, New York, NYU Press, 2015.

13. Albert Moran, Copycat Television: Globalisation, Program Formats and Cultural Identiy, Luton, Bedfordshire, UK, University of Luton Press, 1998; Jean Chalaby, The Format Age: Television's Entertainment Revolution, Cambridge, Polity, 2015; Guillaume Soulez et Kira Kitsopanidou (dir.), « Le levain des médias - Forme, format, média », MEI - Médiation et information, n 39, 2015. 
14. Florent Favard, La promesse d'un dénouement. Énigmes, quêtes et voyages dans le temps dans les séries télévisées de science-fiction contemporaine, thèse de doctorat, Université Bordeaux Montaigne, 2015 ; Florent Favard, Le récit dans les séries de Science-fiction de Star Trek à X-files, Paris, Armand Colin, 2018.

15. Luca Barra, «Master of None, Atlanta, and Audience Engagement in. Contemporary American TV Comedy ", 16:9 Filmtidsskrift, 19 novembre 2017: http://www.16-9.dk/2017/11/master-ofnone/ (consulté le 7 juillet 2019) ; Luca Barra, «Les formes, durées et formats des comédies télévisuelles américaines. Distribution, engagement du public et instabilité des frontières de genre ", dans Marta Boni (dir.), Formes et plateformes de la télévision à l'ère du numérique, Rennes, Presses Universitaires de Rennes, à paraître en 2019.

16. Il faut préciser que cette logique sérialisante - héritée du format de la stand-up comedy dont s'inspire la série Master of None créée et produite par le comédien et comique Aziz Ansari - ne se prolonge pas forcément par la suite, comme c'est le cas de nombreuses séries qui, au fil des saisons, voient leurs arcs narratifs davantage feuilletonner.

17. Alors que la "Quality TV» définit davantage un mode de production luxueux et " cinématique » utilisé par les chaînes américaines câblées pour attirer des publics de niche cultivés et peu coutumiers de la « télévision standard », la « Complex TV » s'applique surtout aux caractéristiques narratives et esthétiques de certaines séries, indépendamment des chaînes qui les produisent puisqu'une série de network peut parfaitement s'inscrire dans le paradigme conceptualisé par Mittell.

18. Warren Buckland (dir.), Puzzle Films: Complex Storytelling in Contemporary World Cinema, Malden, Wiley-Blackwell, 2009.

19. David Bordwell, "What-if Movies: Forking Paths in the Drawing Room », Observations on film art (blog), 2014: http://www.davidbordwell.net/blog/2014/11/23/what-if-movies-forking-pathsin-the-drawing-room/, consulté le 7 juillet 2019; Steffen Hven, Cinema and Narrative Complexity. Embodying the Fabula, Amsterdam, Amsterdam University Press, 2017.

20. Marta Boni, « Mondes sériels et complexité », Écrans, Volume 2, n 4, 2015, p. 55-68.

21. Favard, 2015, p. 32-37.

22. Favard, 2015, p. 36.

23. Raphaël Baroni, La Tension narrative. Suspense, curiosité, surprise, Paris, Seuil, 2007.

24. Alain Boillat, Cinéma, machine à mondes : essai sur les films à univers multiples, Genève, Georg, 2014.

25. Mireille Berton, «Flashback, trauma et répétition narrative dans les séries américaines contemporaines ", Télévision, n 7, 2016, p. 173-189.

26. Plusieurs auteur·e's ont noté également la nécessité de revoir la conceptualisation de l'amateur-trice de séries télévisées qui est qualifiée tantôt de « sériephile » (voir Hervé Glevarec, La sériephilie. Sociologie d'un attachement culturel, Paris, Ellipses, 2012), tantôt de « téléphile » (voir John Caughie, "Telephilia and Distraction : Terms of Engagement », Journal of British Cinema and Television, vol. 3, n 1, janvier 2008, p. 5-18), ou encore de «télé-cinéphile » (voir Daniela Cardini, "Serial Contradictions : The Italian Debate on TV Series", Series. International Journal of TV Serial Narratives, Volume II, $\mathrm{n}^{\circ}$ 1, printemps 2016, p. 47-54: https://series.unibo.it/article/view/6163, consulté le 7 juillet 2019 ; et Daniela Cardini, « Il tele-cinefilo. Il nuovo spettatore della Grande Serialità televisiva ", Between. Rivista dell'Associazione di Teoria e Storia Comparata della Letteratura, Volume 4, n' 8, novembre 2014, p. 1-30:http://ojs.unica.it/index.php/between/article/ view/1361 ?fbclid =IwAR01riiC45RrKUXLIl4lT_Gv0YlPmPclEneLMrMEa1mbG8CmOkh_QkrQ9jY (consulté le 7 juillet 2019).

27. Veronica Innocenti et Guglielmo Pescatore, "Changing Series: Narratives Models and the Role of the Viewer in Contemporary Television Seriality ", Between. Rivista dell'Associazione di Teoria e Storia Comparata della Letteratura, Volume 4, n 8, 2014, p. 1-15 : http://ojs.unica.it/ index.php/between/article/view/4, consulté le 19 juin 2019. 
28. Anne Besson, Constellations. Des mondes fictionnels dans l'imaginaire contemporain, Paris, CNRS Édition, 2015 ; Anne Besson, « De l'ensemble à la totalité : l'effet de monde dans les littératures de l'imaginaire contemporaines", Belphégor, n 14, 2016: http://journals.openedition.org/ belphegor/650, consulté le 7 juillet 2019.

29. Anaïs Goudmand, «Les séries transmédiatiques. Des univers sans fins? ", Proteus, $\mathrm{n}^{\circ} 9$, p. 8-18, 2015 : http://www.revue-proteus.com/articles/Proteus09-1.pdf, consulté le 7 juillet 2019 ; Henry Jenkins, La culture de la convergence : des médias au transmédia, Paris, Armand Colin, 2013 ; Richard Saint-Gelais, Fictions transfuges. La transfictionnalité et ses enjeux, Paris, Seuil, 2011 ; Stéphane Benassi, «Transfictions », MédiaMorphoses, Hors-Série, 2007, p. 158-162.

30. Raphaël Baroni et François Jost (dir.), “Repenser le récit avec les séries télévisées”, Télévision, $n^{\circ}$ 7, 2016 : https://www.cairn.info/revue-television-2016-1.htm, consulté le 19 juin 2019 ; Anaïs Goudmand, "Narratologie du récit sériel. Présentation de quelques enjeux méthodologiques ", Proteus, $n^{\circ}$ 6, 2013, p. 81-89 : http://www.revue-proteus.com/abstracts/06-10.html, consulté le 7 juillet 2019; Monica Michlin, «More, More, More. Contemporary American TV Series and the Attractions and Challenges of Serialization As Ongoing Narrative ", Mise au point, $\mathrm{n}^{\circ}$ 3, 2011: http://map.revues.org/927, consulté le 7 juillet 2019.

31. Robert C. Allen, (dir.), To Be Continued... Soap Operas Around the World, Londres/NewYork, Routledge, 1995; David Buxton, Les séries télévisées. Formes, idéologie et mode de production, Paris, L'Harmattan, 2010.

32. Marta Boni, « Working with Maps: Systems, Networks, Series », in A History of Cinema Without Names II. A Research Project, FilmForum/2015, éd. Diego Cavallotti, Federico Giordano, Leonardo Quaresima, Udine, Mimesis, 2017, p. 69-76.

33. Matthieu Letourneux, Fictions à la chaîne. Littératures sérielles et culture médiatique, Paris, Seuil, 2017 ; Matthieu Letourneux (dir.), «Sérialités », Belphégor, n 14, 2016: http:// journals.openedition.org/belphegor/647, consulté le 7 juillet 2019.

34. Letourneux, 2016, p. 2.

35. Jean-Pierre Esquenazi, Éléments pour l'analyse des séries télévisées, Paris, L'Harmattan, 2017 ; Jean-Pierre Esquenazi, Sociologie des cuvres : de la production à l'interprétation, Paris, Armand Colin, 2007.

36. Letourneux, 2016, p. 2.

37. Jane Feuer, "HBO and the Concept of Quality TV », in Quality TV: Contemporary American Television and Beyond, éd. Janet MacCabe et Kim Akass, Londres, I.B. Tauris, 2007, p. 145-157.

38. Valentina Re, «Beyond the Threshold. Paratext, Transcendence, and Time in the Contemporary Media Landscape", in The Politics of Ephemeral Digital Media: Permanence and Obsolescence in Paratexts, éd. Paolo Noto et Sara Pesce, New York, Londres, Routledge, 2016, p. 6074.

39. Letourneux, 2016, p. 5.

40. Letourneux, 2016, p. 6.

41. Esquenazi, 2007b, p. 87.

42. Edgar Morin, Introduction à la pensée complexe, Paris, Seuil, 2005 [nouv. éd.].

43. Marta Boni, 2015.

44. Yuri Lotman, Universe of the Mind: A Semiotic Theory of Culture, Bloomington, Indiana University Press, 1990, p. 131 (notre traduction).

45. http://www.shotlogger.org/, consulté le 7 juillet 2019.

46. Voir par exemple https://medieninitiative.wordpress.com/2017/02/16/deformativecriticism-at-scms17/

47. http://labotele.com/, consulté le 7 juillet 2019.

48. Franco Moretti, Atlas of the European Novel, 1800-1900, Londres, Verso, 1999; Franco Moretti, «Planet Hollywood», New Left Review, n 9, mai-juin 2001: https://newleftreview.org/II/9/ 
franco-moretti-planet-hollywood, consulté le 7 juillet 2019; Franco Moretti, Graphs, Maps, Trees: Abstract Models for a Literary History, Londres, Verso, 2005.

49. Voir le site Web: «Narrative Ecosystems: Exploring Contemporary Serials Narratives as Complex Ecosystems »: http://www.narrativecosystems.org/wp/ , consulté le 7 juillet 2019.

50. Marta Boni, «Des téléspectateurs géolocalisés ", Sens Public, 15 décembre 2017 : http:// www.sens-public.org/article1283.html, consulté le 7 juillet 2019.

51. Voir aussi Abigail De Kosnik, "Piracy is the Future of Television", Convergence Culture Consortium, 2018, http://convergenceculture.org/research/c3-piracy_future_television-full.pdf, consulté le 7 juillet 2019.

\section{RÉSUMÉS}

Comment étudier les spécificités des séries télévisées qui forment des récits évolutifs, multiformes, ouverts, articulés en plusieurs fragments? Retraçant l'emploi de la notion de complexité avec et au-delà de la narratologie et présentant les défis que pose l'analyse de ces objets, la présente contribution propose de lire les séries télévisées à partir d'une approche interdisciplinaire centrée sur la spatialisation des séries. En effet, en complément de l'analyse textuelle et dans l'absence de sources primaires du côté de l'instance de production (lesquelles s'avèrent la plupart du temps inaccessibles), l'analyste des séries télévisées est amenée à explorer cet espace intermédiaire qui se situe entre la représentation et la réception - un espace complexe et fréquemment investi par les usagers, comme l'a montré le modèle circulaire de la culture participative (Jenkins, 2013).

How to study the specificity of television series building evolving, shapeshifting, open, fragmented narratives? Questioning the use of the notion of complexity within and beyond narratology, this article proposes to read television series through an interdisciplinary perspective focused on spatialization. Indeed, beyond textual analysis and in the absence of primary sources on the production side (sources that more often than not prove inaccessible), the television series scholar is bound to explore the intermediate space between representation and reception - a complex space frequently navigated by users, as shown by the circular model of participatory culture (Jenkins, 2013).

\section{INDEX}

Keywords : spatialization, complexity, participatory culture, narrative

Mots-clés : spatialisation, complexité, culture participative, récit

\section{AUTEURS}

\section{MIREILLE BERTON}

Mireille Berton est maître d'enseignement et de recherche à la Section d'Histoire et esthétique du cinéma de l'université de Lausanne. Ses travaux et enseignements portent sur les rapports entre cinéma et sciences du psychisme, sur l'histoire des imaginaires médiatiques, ainsi que sur 
les enjeux narratifs, culturels et sociaux des séries télévisées. Elle a publié Le Corps nerveux des spectateurs. Cinéma et sciences du psychisme autour de 1900 (2015), et a co-dirigé La Télévision du Téléphonoscope à YouTube. Pour une archéologie de l'audiovision (2009), ainsi qu'un numéro sur les séries télévisées de la revue Décadrages. Cinéma à travers champs (2016).

Mireille Berton is a senior lecturer at the Department of Film Studies of the University of Lausanne (Switzerland). Her research focuses on the relationship between cinema and sciences of "the mind", media technologies and the imaginary, and on the cultural history of spectatorship. She has published Le Corps nerveux des spectateurs. Cinéma et sciences du psychisme autour de 1900 (2015), coedited La Télévision du Téléphonoscope à YouTube. Pour une archéologie de l'audiovision (2009) and an issue on television series for Décadrages. Cinéma à travers champs (2016).

\section{MARTA BON}

Marta Boni est professeure agrégée au Département d'histoire de l'art et d'études cinématographiques de l'Université de Montréal, où elle a fondé le Labo Télé. Elle travaille sur les problèmes théoriques et méthodologiques liés aux phénomènes de type sériel, dont elle explore la complexité narrative et l'entrelacement avec la réception. Elle a publié Romanzo Criminale. Transmedia and Beyond (Ca' Foscari, 2013) ; co-dirigé avec Adrienne Boutang, Barbara Laborde et Lucie Mérijeau Networking Images. Approches interdisciplinaires des images en réseau (Presses de la Sorbonne Nouvelle, 2013) et dirigé World Building. Transmedia, Fans, Industries (Amsterdam University Press, 2017), ainsi que plusieurs chapitres et articles sur ces thèmes.

Marta Boni is an associate professor in Film and Television Studies at the University of Montreal, where she founded the Labo Télé. Her work centers on theoretical and pragmatic issues relating to narrative and semiotic complexity. She published Romanzo Criminale. Transmedia and Beyond (Ca' Foscari University Press, 2013); co-edited with Adrienne Boutang, Barbara Laborde et Lucie Mérijeau Networking Images. Approches interdisciplinaires des images en réseau (Presses de la Sorbonne Nouvelle, 2013) ; edited World-Building. Transmedia, Fans, Industries (Amsterdam University Press, 2017); and published several chapters and articles in scientific journals. 